\title{
Fungal Infections in Gastroenterology
}

\author{
Michael AB Naafs MD* \\ MD, Endocrinologist, Health Consultant at Naafs, International Health Consultancy, Rhodoslaan, Oldenzaal, The Netherlands
}

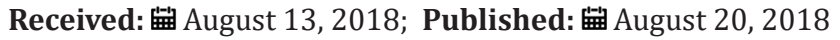

*Corresponding author: Michael AB Naafs, Dutch Internist Endocrinologist, Health Consultant at Naafs, International Health Consultancy, Rhodoslaan 20,7577KN, Oldenzaal, The Netherlands

\begin{abstract}
Fungal infections are increasing in gastroenterology. Cirrhotic patients, liver transplantation recipients, and patients with inflammatory bowel disease (IBD) are vulnerable to these infections. The origin of these fungal infections is frequently the GI tract. The advent of sequencing technology can identify now previously unculturable fungi. Dysbiosis of the mycobiome and microbiome can uncheck normal commensal fungi and turn pathogenic by largely unknown mechanisms Resistance to antifungals is becoming a global problem. New antifungals are in development and they are badly needed. In this mini-review the enteric mycobiota and fungal infections in GI disease are discussed.
\end{abstract}

\section{Introduction}

Fungi are normally present in the gastrointestinal tract as part of the gut microbiome. With the advent of sequencing technology microbes can be studied now that were previous non-culturable. Fungal species, also called the "silent population" are known as the mycobiome [1]. Many pathogenic fungi are commensals in our bodies that cause no harm in normal conditions, but have pathogenic potential. For example, Candida albicans, which causes systemic candidiasis in immunocompromised patients is a normal part of the gut microflora. Why these fungi can turn pathogenic is largely unknown [2]. In a recent editorial in the American Journal of Gastroenterology Brumble and Keaverny pointed to the risky business of fungal infections in patients with cirrhosis, discussing the report of Bajaj et al. [3,4]. Besides liver disease and immunocompromised patients such as in liver transplantation fungal infections occur in patients with inflammatory bowel disease (IBD).In this mini-review the enteric mycobiota and fungal infections in GI disease will be discussed.

\section{The Mycebiome}

Although fungi constitute only a very small part of the gut microbiome, the fungal community (mycobiota) has been shown to contribute actively to GI health and diseases [5-8]. Mycobiome analysis has shown that Candida albicans and Candida parapsilosis constitute most of the fungal community in the human gut $[7,9,10]$. Various studies have confirmed that gut fungal dysbiosis is associated with IBD [11-13]. The abundance of C. albicans, Aspergillus clavatus and Cryptococcus neoformans was noted to be increased in Crohn's disease (CD) patients [14-17]. Most Candida ssp. can also be detected in the feces or intestinal mucosa of patients with ulcerative colitis (UC)- $[18,19]$. To characterize the mycebiota by sequencing variable portions of the fungi genome are targeted, which characterize the fungi to the genes level, such as the 18SrDNA of the fungal internal transcribed spacer (ITS) or the rRNA locus [20]. Each sequencing platform has its strengths and weaknesses in terms of read length, cost and coverage [21-23]. The sequences are grouped into Operational Taxonomic Units (OTUs) and the OTUs are compared with existing databases. The genus abundance is determined from multivariate analysis of the various OTUs. The role of unculturable fungi with unknown taxonomy is not clear and new methods have to be developed to study their interactions within the gut.

A classic way to study interactions between the myco- and microbiome is to introduce dysbiosis in the gut through treatment with antifungals and anti-bacterials [24,25]. Mouse models have been used often in this way to study the gut microbiota. C.albicans is a commensal in the human gut but not in the murine gut. Candida colonization is induced in germ-free, gnotobiotic, infant mice, or conventional mice fed with anti-bacterial and antifungals. this gives some insight into the interrelation between gut bacteria and fungi $[24,25]$. Antibacterial can work to promote or inhibit pathogenic fungal growth [25]. It has been known for more than 30 years that anti-bacterial treatment, notably broad-spectrum antibiotics and antibiotics specific to anaerobic bacteria cause differential effects on fungal susceptibility and predisposes patients to gastrointestinal 
infections by C.albicans [26-28]. Fungi are able to establish a niche in a perturbed gut bacterial microbiome. Studies show that the introduction of small amounts of C.albicans to mice after antibiotic treatment caused significant shifts to the gut microbiota, from phyla to family level, and it may not return to its pre-antibiotic stage in the long-term $[29,30]$. Dysbiosis studies show that the microbiome and commensal fungi interaction is a balancing act. When the microbiome is disrupted, the normally commensal elements in the mycobiome might be unchecked and turn pathogenic.

Foodborne fungi are found in many animal and plant-based foods, surviving the transit through the GI tract and possibly colonizing the gut [31] Hoffmann et al. [32] reported a strong link between consumed foods and fungal abundance in the gut [32] They found an inverse association between Candida and Bacterodes bacteria. Bacteroides was more abundant in high-protein diet users while Candida was more associated with recent carbohydrate consumption. Ultra-processed foods have been linked to a rising prevalence of autoimmune disease [33] It is not known if and how far the mycobiome is involved [33].

\section{Fungi and Liver Disease}

As mentioned before Bajaj et al. [4] studied a cirrhotic cohort of 2743 patients (1691 no infection,918 bacterial and 134 fungal) were included. Patients with fungal infection, all of which were nosocomial, were more likely to be admitted with bacterial infections, on spontaneous bacterial peritonitis prophylaxis, and have diabetes and advanced cirrhosis. Bacterial infection types did not predict risk for fungal infections. Multi-variable analysis showed male gender to be protective, whereas diabetes, longer stay, ICU admission, acute kidney injury (AKI) and admission bacterial infection were associated with fungal infection development (AUC $=0,82$, area under the curve). Fungal infections were associated with significantly higher acute and chronic liver failure (ACLF), inpatient stay, ICU admission, and worse 30-day survival. The case fatality rate was $30 \%$ with most fungal infections but more than $50 \%$ for fungemia and peritonitis. On a multivariable analysis, age, AKI, end stage liver disease, ICU admission and ordinal infection variables impaired survival ( $\mathrm{p}<0,0001 ; \mathrm{AUC}=0,83$ )- (4).

Patients with liver cirrhosis are at an increased risk of fungal infections because the antibiotics used for prevention of spontaneous bacterial peritonitis (SPB) can select for excessive growth of fungi in the intestinal flora with subsequent fungal translocation into the peritoneal cavity and development of spontaneous fungal peritonitis (SFP)- [34,35]. Fungi are much larger than bacteria, which makes fungal translocation along the gut more difficult than in bacterial peritonitis (BT), and may require higher intestinal permeability, which is more common in advanced liver cirrhosis [36,37]. Fungal translocation may be facilitated by upper gastrointestinal bleeding, which is also common in advanced liver cirrhosis [38]. Immunosuppression and malnutrition in liver cirrhosis patients also promote this fungal translocation [35].
Direct percutaneous inoculation of fungi is the proposed route of fungal infection in patients with refractory ascites and a history of paracentesis [38].

SFP is defined as a fungal infection of ascitic fluid with no apparent intra-abdominal source of infection or malignancy [36]. A PMN (polymorph nuclear leukocytes) of $>250$ /cubic $\mathrm{mm}$ in the ascitic fluid with a positive fungal culture regardless of a cocolonization of bacteria is diagnostic of SFB [39]. A positive fungal culture with a PMN count of $<250$ cells.cubic $\mathrm{mm}$ is diagnosed as fungiascites or fungal ascites [39]. Fungal ascites has a higher mortality rate than bacteriascites [39]. Of spontaneous peritonitis cases $0-7,2 \%$ are culture positive for fungi and the most frequent isolate is Candida albicans. Other causative fungi include Candida glabrata, Candida krusei, Cryptococcus ssp. and Penicillium spp. Polymicrobial infections i.e. bacterial co-colonization occurs in $32 \%-74 \%$ of SFP cases, but early diagnosis by conventional microbial culture is difficult because of the time required for growth and the efficiency of PCR or assay of the fungal biomarker 1.3 beta-D-glucan in ascitic fluid has not been established [40-44].

High Child-Pugh or MELD scores increase the risk of SFP in patients with advanced liver cirrhosis [36]. The risk of SFP is increased in patients with liver cirrhosis who undergo invasive procedures [41] and increase with the length of hospital stay $[42,44]$. SFP mortality is estimated to be $56 \%-90 \%[36,39,41]$. Hwang et al. [36] reported a high SFP mortality that was related to unresponsiveness to initial empirical treatment to suspected SBP [36]. SFP is usually diagnosed after the identification of fungi in cultures of ascitic fluid. The mortality is high because of delayed diagnosis, lack of clinical signs, lack of suspicion of SFP and delay in treatment with antifungal therapy [41]. Recent treatment guidelines on management of infections in liver cirrhosis do not include antifungals for prophylaxis or optimum treatment but do include recommendations for fungal infections [34]. Echinocandins are recommended as first-line treatment for patients with liver cirrhosis and nosocomial SFP or critically ill cirrhotic patients with community -acquired SFP [36]. Fluconazole is recommended for less severe infections [41]. However, directed antifungal therapy may not improve the outcome of some patients with SFP because of lack of response to the administration of empirical treatment and delay in antifungal therapy [44]. Although early differentiation between SFP and SBP may be difficult, the clinican is able to suspect SFP or SBP due to multidrug resistance (MDR) if spontaneous peritonitis is not improved after $48 \mathrm{~h}$ empirical antibiotic treatment [39].

\section{Fungal Infections in Liver Transplantation}

Invasive fungal infections (IFIs) are frequent causes of morbidity and mortality in liver transplantation patients. Candidiasis is the most common opportunistic fungal infection of the oral cavity caused by fungi of the genus Candida in immunosuppressed individuals. Bertoncello dos Santos et al. performed a cross 
sectional study in 49 liver transplant recipients at different stages of immunosuppression by the collection of oral samples with a sterile swab [45]. In these 49 patients' samples,39\% had the yeast of the genus Candida Isolated and of these patients, $12 \%$ had candidiasis,60\% of atrophic type and 34\% pseudomembranous. Eleven yeast species were (58\%) Candida non-albicans and eight (42\%) Candida albicans. The high incidence of Candida nonalbicans in the oral cavity with a long history of transplantation warrants a more effective control of health of these patients, the authors concluded [45].

Pasternak et al. investigated the incidence and risk factors of IFIs in pediatric liver transplant recipients during the early post transplantation period ( $<3$ months) - [46]. Data were collected for all pediatric liver transplant recipients recipients registered in a national transplantation center from 2004-2014. Ten IFIs were identified in 81 liver transplant recipients (12,5\%) all occurring during the first month post transplantation. Candida species were responsible for nine cases (90\%) of which four were nonalbicans Candida (44\%). Significant risk factors were multiple blood products transfusion during transplantation, prolonged use of indwelling catheters, prolonged IV antibiotic treatment, surgical complications, pulse steroid treatment and living donor transplantation. The predictive model used two clinical parameters to define high -risk patients: a living donor transplantation and duration of IV antibiotic treatment (area under the ROC curve 0,98).The authors concluded that IFIs are a significant complication occurring in the first month post transplantation and future studies targeting antifungal prophylaxis in these high -risk patients are needed [46]. To date, only small studies and case-series analyzing clinical presentation and risk of IFIs in liver transplantation (LTRs) are available.

Bassetti et al. [47] performed a retrospective multinational study in 10 centers in Europe and Brazil. All consecutive LTRs developing IFI during the period January 2011 to December 2013 were included in the study [47]. A total of 42 LTRs were included. Median age was 52,5 years and $78,6 \%$ of patients were men. Viral hepatitis was the most common cause for liver transplantation $(49,2 \%)$.Candidemia represented the majority of cases $(24,6 \%)$,followed by intra-abdominal candidiasis $(18,4 \% 0$.0verall 30 -day mortality was $23,8 \%$ with higher mortality in patients with candidemia compared with intra-abdominal candidiasis $(37,5 \%$ vs $5,6 \% ; \mathrm{p}=0,02$ ).Multivariate analysis showed candidemia to be a risk factor associated with mortality among LTRs presenting IFIs (odds ratio:11,86;95\% CI 1,5-280; $\mathrm{p}=0.01$ ).Candida albicans represented the most common isolate $(59,5 \%)$.High rates of antifungal resistance were found with $16,7 \%$ and $4,8 \%$ of isolates displaying resistance to azoles and caspfungin respectively [47].

\section{Fungal Infections in IBD}

Patients with inflammatory bowel disease (IBD) are at increased risk of complicatons caused by bacteria, viruses, fungi and parasites. Various factors have been associated with an increased risk of infection in these patients including immunosppressiva and immunomodulatory therapy, severity of disease activity, comorbidity, narcotic analgesic treatment, surgery, malnutrition, leucopenia and older age [48-52]. Stamatiades et al. [53] performed a systematic PubMed search for studies providing data on clinical, microbiology, treatment and outcome of fungal infections in patients with IBD. Fourteen studies with data on 1524 patients were included in the final analysis. The most common fungal infections in patients with IBD were caused by Candida species (903 infections). The most commonly reported site of Candida infection was the GI tract. Available evidence shows that most fungal infections occur within 12 months of IBD treatment and within 6 months when antiTNF-alpha agents are used [53].

Various studies have confirmed that gut fungal dysbiosis is associated with IBD, as mentioned above [11-13]. The abundance of C.albicans, Aspergillus clavatus and Cryptococcus neoformans was increased in Crohn's disease [14-17]. Sokol et al. [54] studied the feacal fungal microbiota in patients with IBD. Bacterial and fungal composition of the microbiota of 235 patients with IBD and 36 healthy subjects (HS) was determined using 16 S and ITS2 sequencing, respctively. The obtained sequences were analysed using the Qime pipeline to assess composition and diversity. The authors observed that fungal microbiota is skewed in IBD, with an increased Basidomycota/Ascomycota ratio, a decreased proportion of Saccharomyces cerviciae and an increased proportion of Candida albicans compared with HS. They also identified disease specific alterations in diversity, indicating that a Crohn's specific gut environment may favour fungi at the expense of bacteria. The concomitant analysis of bacterial and fungal microbiota showed a dense and homogenous correlation network in HS but a dramatically unbalanced netwok in IBD, suggesting the existence of disease specific inter-kingdom alteration [54].

Inter-kingdom alteratons between fungi and bacteria at the gut may also be implicated in the gut mycobiome-gut-brain-axis (GBA). These alterations influence extraintestinal immune responses. Dectin-1 is one of the most important pattern recognition receptos (PRRs) expressed by immune cells that interact with fungal betaglucan.Dectin-1 knockout mice have severe colitis [55]. Fungi are able to synthesize and release neurotransmtters, similar to many bacteria. S.cervisiae and Penicillium chrysogenum can produce high concentrations of norepinephrine. C.albicans is able to produce histamine, another neuromediator involved in appetite regulation, sleep-wake rhythm and cognitive activity [56-58].

Sivamaruthi reviewed the clinical outcome of probiotic and synbiotic therapy for inflammatory bowel diseases. The studies suggested that the intervention of multistrain probiotic preparation or symbiotic preparation performed better than single strain therapy. Moreover, several studies were conducted with VSL\#3 and proved that VSL\#3 was safe and recommended by many clinicians to support the remission of UC, and for maintenance therapy. Most 
of the clinical trials were conducted with a minimum number of patients on short treatment duration or no proper follow-up. Apart from the formulation of best probiotic or symbiotic preparation, dosage, duration, mode of intervention and form of formulation are playing a critical role in the outcome of a clinical trial. Future studies are required to select the probiotic strain or combinations to treat or control IBD [59].

\section{Antifungal Resistance and Candida Auris}

Antifungal resistance is becoming a global problem [60]. Only a few classes of antifungal drugs are available, so the emergence of resistance to single drug classes and now multidrug resistance is a serious problem in patient management. Azole resistance among Candida and Aspegillus species is one of the greatest challenges, followed by echinocandin among some Candida species, especially Candida glabrata. The spread of agriculturally derived azole-resistant Aspergillus fumigatus and emerging threats such as Candida auris are alarming. The molecular mechanisms that cause drug resistance are naturally occurring in less susceptible species and are acquired in strains of susceptible organisms. Drug resistance mechanisms include altered drug interactions, reduced cellular drug concentrations mediated by drug efflux transporters and permeabilty barriers associated with biofilms. Although C.auris is inherently multidrug resistant, other strains typically develop resistance through stepwise selection of multiple drug resistance mechanisms.

The GI tract is a major source of echinocandin drug resistance. Candida species are a part of the human microbiome and can cause systemic infection upon immune suppression. Candida glabrata infections are increasing and have greater rates of antifungal resistance than other species. Healy et al. showed that C.glabrata echinocandin resistance can arise within the GI tract in a murine model and that resistant mutants can readily disseminate upon immune suppression [61]. Various investigational antifungals are currently in preclinical or clinical development, including several of them that have the potential to overcome resistance observed against the azoles and echinocandins. These include agents that also target ergosterol and beta-glucan biosynthesis, as well as compounds with novel mechanisms of action that may also overcome the limitations of currently available antifungal classes, including both resistance and adverse effects toxicity [62].

\section{Conclusion}

Fungal infections are increasing in gastroenterology. Cirrhotic patients, liver transplant recipients and patients with inflammatory bowel disease (IBD) are vulnerable to these infections. The origin of these infections is frequently the GI tract. The advent of sequencing technology can identify now previously unculturable fungi. Dysbiosis of the mycobiome and microbiome can uncheck normal fungal commensals and turn them pathogenic by largely unknown mechanisms. Antibiotics can work to promote or inhibit pathogenic fungal growth. Dysbiosis can also be induced by diet and foodborne pathogens, including fungi.
The mortality of fungal infections in patients with acute and chronic end-stage liver cirrhosis is high $(>50 \%)$ in cases of spontaneous fungal peritonitis (SFP). Age, acute kidney injury (AKI), end -stage liver disease, ICU admission and ordinal infection variables all impair survival. Invasive fungal infections (IFIs) are frequent causes of morbidity and mortality in liver recipients' patients. Significant risk factors were multiple blood products transfusions during liver transplantation, prolonged use of indwelling catheters, pulse steroid treatment and living donor transplantation. Various studies have confirmed that gut dysbiosis is associated with IBD. Fungal microbiota is skewed in IBD patients and Crohn's specific gut microenvironment may favor fungi at the expense of bacteria. Antifungal resistance is becoming a global problem. Various investigational antifungals are currently in preclinical and clinical development. They are badly needed to meet the challenge of increasing fungal infections in gastroenterology.

\section{References}

1. Gillevet PM, Sikaroodi M, Torzilli AP (2009) Analyzing salt-marsh fungal diversity: Comparing arisa fingerprinting with clone sequencing and pyrosequencing. Fungal Ecol 2(4): 160-167.

2. Drgona L, Khachatryan A, Stephens J (2014) Clinical and economic burden of invasive fungal diseases in Europe: focus on pre-emptive and empirical treatment of Aspergillus and Candida species. Eur J Clin Microbiol Inf Dis 33(1): 7-21.

3. Brumble L, Keaverny AP (2018) Editorial: The Risky Business of Fungal Infections in Patients with Cirrhosis. Am J Gastroenterol 113: 564-566.

4. Bajaj JS, Reddy KR, Tandon P (2017) Prediction of fungal infection development and their impact on survival using the NACSELD cohort. Am J Gastroenterol 113(4): 556-563.

5. Lucas Lopez R, Grande Burgos MJ, Galvez A (2017) The human gastrointestinal tract and oral microbiota in inflammatory bowel disease: a state of the science review. APMIS 125: 3-10.

6. Ott SJ, Kuhbacher T, Musfeldt M (2008) Fungi and inflammatory bowel diseases: alleviations of composition and diversity. Scand J Gastroenterol 43: 831-841.

7. Mukherjee PK, Sendid B, Hoarau G (2015) Mycebiota in gastrointestinal diseases. Nat Rev Gastroenterol Hepatol 12: 77-87.

8. Wang ZK, Yang YS, Stefka AT (2014) Review article: fungal microbiota and digestive diseases. Aliment Pharmacol Ther 39: 751-66.

9. Scanlon PD, Marchesi JR (2008) Micro-eukaryotic diversity of the human distal gut microbiota: qualitative assessment using culture-dependent analysis of faeces. ISME J 2: 1183-1193.

10. Tang J, lliev D, Brown J (2015) Mycebiome: approaches to analysis of intestinal fungi. J Immunol Methods 421: 112-121.

11. Moyes DL, Naglik JR (2012) The mycebiome: influencing IBD severity. Cell Host Microbe 11: 551-552.

12. Iliev ID, Funari VA, Taylor KD (2012) Interactions between commensal fungi and the C-type lectin receptor Dectin-1 influence colitis. Science 336: 1314-1317.

13. Richard ML, Lamas B, Liguori G (2015) Gut fungal microbiota: the Yin and Yang of inflammatory bowel disease. Inflamm Bowel Dis 21: 656665.

14. Li Q, Wang C, Tang C (2014) Dysbiosis of gut fungal microbiota is associated with mucosal inflammation in Crohn's disease. J Clin Gastroenterol 48: 515-523. 
15. Standaert Vitse A, Joualt T, Van derWalle P (2006) Candida albicans is an immunogen for anti-Saccharomyces cerevisae antibody markers of Crohn's disease. Gastroenterology 130: 1764-1775.

16. Standaert Vitse A, Sendd B, Joossens M (2009) Candida albicans colonization and ASCA in familial Crohn's disease. Am J Gastroenterol 104: $1745-1753$.

17. Liguori G, Lamas B, Richard ML (2016) Fungal dysbiosis in mucosaassociated microbiota of Crohn's disease patients. J Crohns Colitis 10: 296-305.

18. Zwolinska Woislo M, Brzozowsk T, Budoh A (2009) Effect of Candida colonization on human ulcerative colitis and the healing of inflammatory changes of the colon in the experimental model of colitis ulcerosa. J Physiol Pharmacol 60: 107-118.

19. Ksiadzyna D Semianow Wejchert J, Nawrot U (2009) Serum concentration of interleukin 10..anti-mannan Candida antibodies and the fungal colonization of the gastrointestinal tract in patients with ulcerative colitis. Adv Med Sci 54: 170-176.

20. Suhr Mj,Hullen Adams HE (2015) The hman gut mycobiome: Pitfalls and potentials-A mycologist's perspective. Mycologia 107: 1057-1073.

21. Weinstock GM (2012) Genomic approaches to studying the human microbiota. Nature 489: 250-256.

22. Kuczynski J, Lauber CL, Walters WA (2012) Experimental and analytical tools for studying he microbiome. Nat Rev Genet 1: 47-58.

23. DiBella JM, BaoY, Gloor GB (2013) High throughput sequencing methods and analysis for microbiome research. J Microbiol Methods 95: 401-414.

24. Mc Frederick QS, Mueller UG, James RR (2014) Interactions between fungi and bacteria influence microbial community structure in the megachile rotundata larval gut. Proc R Soc London B 281(1779): 20132653.

25. Azevedo MM, Texeira Santos R, Silva AP (2015) The effect of antibacterial and non-antibacterial compounds alone or associated with antifungals upon fungi. Front Microbiol 6: 669.

26. Kennedy MJ (1989) Regulation of Candida albicans populations in the gastrointestinal tract: mechanisms and significance in GI and systemic candidiasis. Curr Top Med Mycol 3: 3125-3402.

27. Clark JD (1971) Influence of antibiotics or certain intestinal bacteria on orally administered candida albicans in germ-free and conventional mice. Infect Immun 4: 731-737.

28. Samonis G, Gikas A, Anaissie EJ (1993) Prospectve evaluation of broadspectrum antibiotics on gastrointestinal yeast colonization of humans. Antimicr Agents Chemother 37: 51-53.

29. Erb Downward JR, Falkowski NR, Mason KL (2013) Mobilisation of post-antibiotic bacterial community reassembly and host response by candida albicans. Sci Rep 3: 2191.

30. Mason KL, Erb Downward JR, Mason Kd (2012) Candida albicans and bacterial microbiota interaction in the cecum during recolonization following broadspectrum antibiotic therapy. Infect Immun 80: 33713380 .

31. David LA, Maurice CF, Carmody RN, Gootenberg DB, Button JE, et al (2014) Diet rapidly and reproducibly alters the human gut microbiome. Nature 505(7484): 559-563.

32. Christian Hoffmann, Serena Dollive, Stephanie Grunberg, Jun Chen, Hongzhe Li, et al. (2013)Archaea and fungi of the human gut microbiome; correlations with diets and bacterial residents. Plos One 8(6): e66019.

33. Naafs MA (2018) Ultra-Processed Foods, Gut Health, Autoimmunity and Diets. CPQ Medicine 2(2): 01-12.

34. Nadim MK, Durand F, Kellum JA, Levitsky J, O Leary JG, et al. (2016) Management of the critically ill patient with cirrhosis:A multidisciplinary perspective. J Hepatol 64(3): 717-735.
35. Fiore M, Leone S (2016) Spontaneous fungal peritonitis. Epidemiology, current evidence and future prospective. World J Gastroenterology 22(34): 7742-7747.

36. Hwang SY, Yu SJ, Lee JH, Kim JS, Yoon JW, et al. (2014) Spontaneous fungal peritonitis: a severe complication in patients with advanced liver cirrhosis. Eur J Clin Microbiol Infect Dis 33(2): 259-264.

37. Bremmer DN, Garavaglia JM, Shields RK, (2015) Spontaneous fungal peritonitis: a devastating complications of cirrhosis. Mycoses 58(7): 387-393.

38. Hassan EA, Abd El Rehim AS, Hassany SM, Ahmed AO, Elsherbiny NM, et al. (2014) Fungal infections in patients with end-stage liver disease: low frequency or low index of suspicion. Int J Infect Dis 23: 69-74.

39. Fiore M, Maraolo AE, Leone S, Gentile I, Cuomo A, et al. (2017) Spontaneos peritonitis n critically ill cirrhotic patients: a diagnostic algorithm for clinicians and future perspectives. Ther Clin Rsk Manag 13: 1409-1414.

40. Fiore M, Leone S (2016) Use of antifungals in critically ill cirrhotic patients with spontaneous peritonitis. J Heptanol 64(4): 986-987.

41. Gravito Soares M, Gravito Soares E, Lopes S, Ribeiro G, Figueiredo P (2017) Spontaneous fungal peritonitis: a rare but severe complicaton of liver cirrhosis. Eur J Gastroenterol Heatl 29(9): 1010-1016.

42. Bucsics T, Schwabl P, Mandorfer M, (2016) Prognosis of cirrhotic patients with fungiascites and spontaneous fungal peritonitis (SFP) Hepatol 64(6): 1452-1454.

43. Eikhateef S, Gouda NS, Ibrahim AS, Rokiah A, Laila E (2017) PCRdetected fungal infection is associated with fatal outcomes in cirrhotic patients with spontaneous peritonitis. EJBAS 4(1): 42-46.

44. Fiore M, Chiodini P, Pota V, Sansone P, Passavanti MB, et al. (2017) Risk of spontaneous fungal peritonitis in hospitalized cirrhotic patients with ascites: a systematic review of observational studies and meta-analysis. Minerva Anestesiol 83(12): 309-316.

45. Santos SBD, Sabadin CES, Mario DN, Rigo L, Barbosa DA (2018) Presence of Candida spp. and candidiasis in liver transplant patients. An Bras Dermatol 93(3): 356-361

46. Pasternak Y, Rubin S, Bilavsky E, Mozer Glassberg Y, Levy, et al. (2018) Risk factors for early invasive fungal infections in paediatric liver transplant recipients. Mycoses.

47. Bassetti M, Peghin M, Carnelutti A, Righi E, Merelli M, et al. (2017) Invasive Candida Infections in Liver Transplant Recipients:Clinical Features and Risk Factors for Mortality. Transplant Direct May 3(5): e156.

48. Viget N, Vernier Massouile G, Salmon Ceron D (2007) Oppportunistic infections in patients with inflammatory bowel disease:prevention and diagnosis. Gut 57(4): 549-558.

49. Rahier JF, Magro F, Abreu C, Armuzzi A, Ben Horin S, et al. (2014) Second European evidence-based consensus on the prevention,diagnosis and management of opportunistic infections in inflammatory bowel disease. J.Crohns Colitis 8(6): 443-468.

50. Lichtenstein GR, Feagan BG, Cohen RD, Salzberg BA, Diamond RH, et al. (2012) Serious infection and mortalty in patients with Crohn's disease: more than 5 years follow-up in the TREAT registry. Am J Gastroenterol 107(9): 1409-1422.

51. Toruner M, Loftus EV Jr, Harmsen WS, Zinsmeister AR, Orenstein R, et al. (2008) Risk factors for opportunistic infection in patients with inflammatory bowel disease. Gastroenterology 134(4): 929-936.

52. Bonovas S, Fiorino G, Allocca M, Lytras T, Nikolopoulos GK, et al. (2016) Biologic therapies and risk of infection and malignancy in patients with inflammatory bowel disease; a systematic review and network metaanalysis. Clin Gastroenterol Hepatol 14(10)1385-1397: e10.

53. Stamatiades GA, Ioannou P, Petrikkos G, Tsioutis C (2018) Fungal infections in patients with inflammatory bowel disease: A systematic review. Mycoses 61(6): 366-376. 
54. Sokol H, Leducq V, Aschard H, Pham HP, Jegou S, et al. (2016) Fungal microbiota dysbiosis in IBD. Gut 66(6): 1039-1048.

55. Brown GD, Gordon S (2003) Fungal beta-glucans and mammalian immunity. Immunity 19(3): 311-315.

56. Tsavkelova EA, Botvinko, Kudrin VS, Oleskin AV (2000) Detection of neurotransmitter amines in microorganisms with the use of highperformance liquid Dokl Bio chem Proc Acad. Sci USSR Bio chem Sci 372(1-6): 115-117.

57. Naafs MA (2018) Microbial Endocrinology in Microbiology.Int.J.Clin. Endocrinol. 2(1): 004-010.

58. Enaud R, Vandenborght L, Coron N, , Bazin T, Prevel R, et al. (2018) The Mycobiome: A Neglected Component in the Microbiota-Gut-Brain-Axis. Microorganisms 6(1).

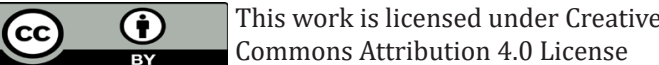

To Submit Your Article Click Here: Submit Article

DOI: $10.32474 /$ CTGH.2018.01.000114
59. Sivamarathi B (2018) A comprehensive review on clinical outcome of probiotic and synbiotic therapy forr inflammatory bowe disease. Asian Pac J Trop Biomed 8(3): 179-186.

60. Perlin DS, Richardson RR, Izquierido AA (2017) The global pattern of antifungal resistance: prevalence,mechanisms and management. The Lancet Inf.Dis. 17(12): e383-e392.

61. Healey KR, Nagasaki Y, Zimmerman M, Kordalewska M, Park S, et al. (2017) The Gastrontestinal Tract is a Major Source of Echinocandin Drug Resistance in a Murine Model of Candida glabrata Colonization and Systemic Dissemination. Antimicrobe Agents Chemother 61(12): 14121417.

62. Wiederhold NP (2017) Antifungal resistance: current trends and future strategies to combat. Inf Drug Resist 10: 249-259.

$\begin{gathered}\text { Current Trends in Gastroenterology } \\ \text { and Hepatology }\end{gathered}$
Assets of Publishing with us
- Global archiving of articles
- Immediate, unrestricted online access
- Rigorous Peer Review Process
- Authors Retain Copyrights
- Unique DOI for all articles

